\title{
A NOTE ON MODEL COMPLETE MODELS AND GENERIC MODELS ${ }^{1}$
}

\author{
SAHARON SHELAH
}

ABSTRACT. We prove that there are many maximum model complete (= generic) models, and that there exists an (uncountable) theory with no generic models.

After Barwise and Robinson [1] we say a model $M$, of a (first-order) theory $T$, completes $T$ if every extension of $M$, which is a model of $T$, is an elementary extension of $M$. (By [1, Theorem 3.4, p. 129], $M$ completes $T^{f}$ iff it is $T$-generic.) It is known

LEMMA 1. If $M$ completes $T$ and $N$ is an elementary submodel of $M$, then $N$ also completes $T$ (it follows from Theorem 1.2).

For a cardinal $\lambda$ let $M c(\lambda)$ be the least cardinal $\kappa$, such that for all $T$ of power $\leqq \lambda$, if $T$ is completed by some model of power $\kappa$, then for all $\mu \geqq \lambda$ there is a model which completes $T$ and whose power is $\geqq \mu$.

THEOREM 2. $M c(\lambda)=\mu_{\lambda}$ (=the Hanf number of omitting a type).

REMARK. For the values of $\mu_{\lambda}$ see, e.g., Chang [2, $\$ 2$, p. 47]; he denotes $\mu_{\lambda}$ by $m_{\lambda}$.

THEOREM 3. For arbitrarily large cardinals $\kappa$ smaller than the first measurable cardinal there exists a complete and countable $T$ and a model $M$ of power $\kappa$ which complete $T$, and no proper extension of $M$ completes $T$.

Answering Question 8.1 of [1] we prove in $\$ 2$ :

THEOREM 4. There is an uncountable theory $T$ with no T-generic model.

(This was also proved, independently, by P. Henrard, and later by Macintyre.) Only in $\$ 2$ knowledge of [1] is assumed.

Notation. $|M|$ is the universe of the model $M .|A|$ is the cardinality of the set $A$ (so $|L|$ is the number of formulas of $L$ ). $\|M\|$ is the cardinality of (the universe of) $M$. Infinite cardinals are denoted by $\lambda, \mu, \kappa$.

Received by the editors May 25, 1971 and, in revised form, November 5, 1971.

AMS 1970 subject classifications. Primary 02H05.

Key words and phrases. Generic models, model complete, omitting types, maximal models.

${ }^{1}$ The preparation of this paper was sponsored in part by NSF Grants GP-22937 and GP-27964.

(c) American Mathematical Society 1972 
1. THEOREM 1.1.2 Let $T$ be a theory, $p$ a type in a language $L$, and $M$ an infinite model of $T$ which omits $p$. Then there are a language $L_{1}$, a theory $T_{1}$ and a type $p_{1}$ in $L_{1}$ and a model $M_{1}$ of $T_{1}$ which omits $p_{1}$ such that:

(a) $\left|L_{1}\right| \leqq|L|+\aleph_{0}, T_{1}$ is complete.

(b) $M_{1}$ completes $T_{1}$, it omits $p_{1}$ and $\left\|M_{1}\right\|=\|M\|$.

(c) A model of $T_{1}$ completes $T_{1}$ iff it omits $p_{1}$.

(d) If every extension of $M$ which is a model of $T$ realizes $p$ then no extension of $M_{1}$ completes $T_{1}$.

(e) If $T$ has no model of cardinality $\lambda$ which omits $p$ then there is no model which completes $T_{1}$ in cardinality $\lambda$.

Proof. Without loss of generality assume in $L$ there are no function symbols. Let $p=\left\{\phi_{i}(x): i<|p|\right\}$. Let us choose infinite disjoint subsets of $|M|, A_{i}, i<|p|$, such that $|M|=\bigcup A_{i}$.

We expand $M$ to a model $M^{1}$ by adding the following relations:

(1) $P_{i}^{M M^{1}}=A_{i}$ for every $i<|p|$ (i.e., $P_{i}^{M^{1}}$ is a one-place relation, and $P_{i}$ the corresponding predicate).

(2) A relation $R^{M^{1}}$ such that $\langle a, b\rangle \in R^{M^{1}}$ iff there is $i<|p|, b \in P_{i}^{M^{1}}$, $M \vDash \neg \phi_{i}(a)$ and for every $j<i, M \vDash \phi_{j}[a]$. $P_{i}^{M M^{1}}$.

(3) An equivalence relation $E_{1}^{M^{1}}$ such that: $a E_{1}^{M^{1}} b$ iff for some $i, a, b \in$

Now let us define a model $M^{2}$. Its set of elements is

$$
\left|M^{2}\right|=\left\{\langle a, \alpha\rangle: a \in\left|M^{1}\right|, \alpha \leqq \omega\right\} .
$$

Its relations and functions are:

(4) An equivalence relation $E_{2}^{M^{2}}$ such that:

$$
\langle a, \alpha\rangle E_{2}^{M I^{2}}\langle b, \beta\rangle \text { iff } a=b .
$$

(5) For every $n$-place relation $Q^{M^{1}}$ let

$Q^{M^{2}}=\left\{\left\langle\left\langle a_{1}, \alpha_{1}\right\rangle, \cdots,\left\langle a_{n}, \alpha_{n}\right\rangle\right\rangle:\left\langle a_{1}, \cdots, a_{n}\right\rangle \in Q^{M I^{1}} ; \alpha_{1}, \cdots, \alpha_{n} \leqq \omega\right\}$.

(6) An equivalence relation $E_{3}^{M M^{2}}$ such that $\langle a, \alpha\rangle E_{3}^{M M^{2}}\langle b, \beta\rangle$ iff

(a) $a=b$,

(b) $\alpha=\beta$ or $\alpha=2 n+1, \beta=2 n$ or $\alpha=2 n, \beta=2 n+1$.

(7) For every $i<|p|$ a function $F_{i}^{M M^{2}}(x, y)$ such that for every $a, b \in\left|M^{2}\right|$ :

(a) if $M^{2} \vDash \neg a E_{2} b \vee \neg P_{i}(a)$ then $F_{i}^{M M^{2}}(a, b)=a$,

(b) if $M^{2} \vDash a E_{2} b \wedge P_{i}(a)$ then $M^{2} \vDash P_{i}\left(F_{i}(a, b)\right)$,

(c) if $M^{2} \vDash a E_{2} b \wedge a E_{2} c \wedge P_{i}(a) \wedge b \neq c$ then $M^{2} \vDash \neg E_{2}\left(F_{i}(a, b), F_{i}(a, c)\right)$.

\footnotetext{
${ }^{2}$ Added IN PROOF (May 11, 1972). In Theorem 1.1 if $p$ is countable, we can define $M_{1}$ so that $M \equiv N$ implies $M_{1} \equiv N_{1}$. This may help to improve Theorem 3 .
} 
Now we define the $M_{1}$ we wanted as an expansion of $M^{2}$ by:

(8) For every $n<\omega, i_{1}, \cdots, i_{n}<|p|$ (not necessarily distinct) and formula $\phi\left(x_{1}, \cdots, x_{n}\right)$ of the language of $M^{2}$, we add the relation $R_{\phi, i_{1}}^{M_{1}}, \cdots, i_{n}$ defined by

$$
R_{\phi, i_{1}}^{M_{1} \cdots, i_{n}}=\left\{\left\langle a_{1}, \cdots, a_{n}\right\rangle: M^{2} \vDash\left[P_{i_{1}}\left(a_{1}\right) \wedge \cdots \wedge P_{i_{n}}\left(a_{n}\right) \wedge \phi\left(a_{1}, \cdots, a_{n}\right)\right]\right\} .
$$

Now let $T_{1}$ be the theory of $M_{1}, L_{1}$ its language, and $p_{1}=\left\{\neg P_{i}(x): i<|p|\right\}$. Let us now prove that:

(*) A model of $T_{1}$ completes $T_{1}$ iff it omits $p_{1}$.

By (8) it is clear that every model of $T_{1}$ which omits $p_{1}$ completes $T_{1}$. Suppose now $N$ is a model of $T_{1}$ which realizes $p_{1}$, and let $a \in|N|$ realize $p_{1}$. As $N$ is a model of $T_{1}$, by (4) and (6), there are distinct elements $c, b_{n}, 0 \leqq n<\omega$, such that:

$$
\begin{aligned}
& N \vDash b_{2 n} E_{3} b_{2 n+1}, \quad N \vDash(\forall x)\left(x E_{3} c \rightarrow x=c\right), \\
& \left.N \vDash a E_{2} b_{n} \text { and } \quad N \vDash a E_{2} c \quad \text { (for every } n\right) .
\end{aligned}
$$

We now define now a submodel $N_{1}$ of $N$, whose set of elements is $\left|N_{1}\right|=$ $|N|-\left\{c, b_{0}\right\}$. Now $N_{1}$ is not an elementary submodel of $N$ because

$$
N_{1} \vDash(\forall x)\left(x E_{3} b_{1} \rightarrow x=b_{1}\right), \quad N \vDash(\exists x)\left(x E_{3} b_{1} \wedge x \neq b_{1}\right) .
$$

On the other hand $N, N_{1}$ are isomorphic: define $F$ by:

$$
F(c)=b_{1} F\left(b_{n}\right)=b_{n+2} \quad(\text { for } 0 \leqq n<\omega)
$$

and

$$
F\left(a^{1}\right)=a^{1} \quad \text { for } a^{1} \in N-\left\{c_{1} b_{1}, b_{2} \cdots\right\} .
$$

Clearly, $F$ is an isomorphism from $N$ onto $N_{1}$.

So $N, N_{1}$ are models of $T, N_{1}$ does not complete $T$, hence also $N$ does not complete $T$. So we proved $(*)$.

Now (a) is immediate; (b) follows from the definition of $\left|M^{2}\right|=\left|M_{1}\right|$ and $\left({ }^{*}\right) ;(c)$ is $\left(^{*}\right)$; (d) is clear from $\left({ }^{*}\right)$ and $(2)$; and for (e) we should notice also (7) (which implies that if $N$ is a model of $T_{1}$, which omits $p_{1}$, then $\|N\|$ is equal to the number of $E_{2}^{N}$-equivalence classes in $\left.|N|\right)$. So we prove the theorem.

The following theorem was already known to Robinson:

THEOREM 1.2. For every theory $T$ there is a set $P$ of types (not all 1-types necessarily) such that: any model $M$ completes $T$ if and only if $M$ is a model of $T$ omitting every type $p \in P$, and $|P| \leqq|T|+\aleph_{0}$.

Proof. Let $M$ be a model, and $|M|=\left\{a_{i} \mid i<\alpha\right\}$ and Diag $M$ be the set of sentences $\phi\left(a_{i}, \cdots, a_{n}\right)$ which are satisfied by $M$ where $\phi$ is a basic formula ( $=$ an atomic or negation of an atomic formula). Clearly, 
$M$ completes $T$ if and only if $T \cup \operatorname{Diag} M$ is a complete theory. By the compactness theorem, this implies: $M$ completes $T$ if and only if: for every formula $\phi\left(x_{1}, \cdots, x_{m}\right)$ and elements $b_{0}^{1}, \cdots, b_{m}^{0} \in|M|$, there are $\phi_{1}\left(b_{1}^{1}, b_{2}^{1}, \cdots\right), \cdots, \phi_{n}\left(b_{1}^{n}, b_{2}^{n}, \cdots\right)$ in Diag $M$ such that

$$
T \cup\left\{\phi\left(b_{1}^{1}, \cdots\right), \cdots, \phi_{n}\left(b_{1}^{n}, \cdots\right)\right\} \vdash \phi\left(b_{1}, \cdots, b_{n}\right)
$$

or, equivalently,

$$
T \vdash\left(\forall \cdots x_{j}^{i} \cdots\right)\left[\bigwedge_{i} \phi_{i}\left(x_{1}^{i} \cdots\right) \rightarrow \phi\left(x_{1}^{0}, \cdots\right)\right]
$$

(we should identify the variables $x_{k}^{i}, x_{e}^{k}$ if $a_{i}^{k}=a_{e}^{k}$ ). For every formula $\phi=\phi\left(x_{1}, \cdots, x_{n}\right)$ let $\Gamma_{\phi}$ be the set of formulas $\theta\left(x_{1}, \cdots, x_{n}, \cdots, x_{m}\right)$ which are conjunctions of basic formulas and

$$
T \vdash\left(\forall x_{1}, \cdots, x_{m}\right)\left[\theta\left(x_{1}, \cdots, x_{m}\right) \rightarrow \phi\left(x_{1}, \cdots, x_{n}\right)\right] .
$$

Let $p_{\phi}=\left\{\neg\left(\exists x_{n+1}, \cdots, x_{m}\right) \theta\left(x_{1}, \cdots, x_{n}, \cdots, x_{m}\right): \theta \in \Gamma_{\phi}\right\}$. Clearly, $M$ completes $T$ if and only if for every $\phi, T$ omits $p_{\phi}$. So $P=\left\{p_{\phi} \mid \phi\right.$ a formula\} satisfies the condition of the theorem.

Proof OF THEOREM 2. By the definitions of $M c(\lambda), \mu_{\lambda}$, clearly Theorem 1.1 implies $M c(\lambda) \geqq \mu_{\lambda}$. Suppose that $M$ completes $T,\|M\| \geqq \mu_{\lambda}, \lambda \geqq|T|$. So by $1.2, M$ is a model of $T$ and omits every $p \in P$. By, e.g., Chang [2, p. 47, (D)], the Hanf number for a sentence in $L_{\lambda^{+}, \omega}$ is $\mu_{\lambda}$, and clearly being a model of $T$ omitting every $p \in P$ can be expressed in $L_{\lambda^{+}, \omega}$. So $T$ has arbitrarily large models omitting every $p \in P$, hence by 1.2 arbitarily large models completing $T$. This means $M c(\lambda) \leqq \mu_{\lambda}$. So $M c(\lambda)=\mu_{\lambda}$.

Proof of TheOrem 3. This can be proved using 1.1 and the following (see Malitz and Reinhart [4, Theorem XX]).

THEOREM. For arbitrarily large cardinals $\lambda$ smaller than the first measurable cardinal, there is a model $M_{\lambda},\left\|M_{\lambda}\right\|=\lambda$, with countable type and with $a$ one place relation $P, P^{M I_{\lambda}}=\left\{c_{n} \mid c_{n}<\omega\right\}$, such that: for no proper extension $N$ of $M_{\lambda}$ which is elementarily equivalent to $M_{\lambda}, P^{N}=P^{M_{\lambda}}$.

(For characterization of those $\lambda$ which satisfy this, see [4].)

2. Let $N$ be the standard model of natural numbers with addition, multiplication and individual constant $m$ for each natural number $m$. Let $T=T h(N)$, and the language be $L^{*}$. Let $K=T \cup\left\{c_{i} \neq c_{j}: i<j<\aleph_{1}\right\}$, and its language $L, K_{1}=T \cup\left\{c_{i} \neq c_{j}: i<j<\omega\right\}$ and its language $L_{1}$.

THEOREM 2.1. There is no K-generic model. 
Proof. It is easy to check that $P\left(c_{1}, \cdots, c_{m}, a_{1}, \cdots, a_{m}\right)$ for both $K$ and $K_{1}$ is a forcing condition iff

$$
\begin{aligned}
\left(\exists x_{1} \cdots\right)\left(\exists y_{1} \cdots\right)\left[P\left(x_{1}, \cdots, y_{1}, \cdots\right) \wedge \bigwedge_{i \neq j} x_{i} \neq x_{j}\right] \in T \\
\left(a_{1}, \cdots,- \text { new constants }\right) .
\end{aligned}
$$

Let $\Gamma$ be the set of formulas $\phi\left(x_{1}, \cdots, x_{n}\right)$ in $L^{*}$ such that for any distinct natural numbers $m_{1}, \cdots, m_{n} N \vDash \phi\left[m_{1}, \cdots, m_{n}\right]$.

We shall prove now

$$
K_{1}^{f}=T \cup\left\{\psi\left(c_{i 1}, \cdots, c_{i_{n}}\right): i_{1}, \cdots, i_{n}\right. \text { are distinct }
$$

$$
\text { and }\left\langle\omega, \psi\left(x_{1}, \cdots, x_{n}\right) \in \Gamma\right\} \text {. }
$$

Construction. Let $A$ be a countable set of new individual constants, let $P$ be a forcing condition. We shall show that there is a $K_{1}$-generic model, which is a model of $K_{1}^{f}(P)=\left\{\phi \in L(A): P \Vdash^{*} \phi\right\}$ and whose reduct to $L^{*}$ is $N$. Let $\left\{\phi_{i}: i<\omega\right\}$ be the set of sentences of $L(A), A=\left\{a_{n}: n<\omega\right\}$. We define by induction $P_{n}$ :

(1) $P_{0}=P$.

(2) If $P_{3 n}$ is defined, then there is a $Q \supset P_{3 n}$, such that $Q \Vdash \phi_{n}$ or $Q \Vdash \neg \phi_{n}$. Let $P_{3 n+1}=Q$.

(3) If $P_{3 n+1}$ is defined it is easy to see that there is a natural number $m$ such that $P_{3 n+1} \cup\left\{c_{n}=m\right\}$ is a forcing condition. Let $P_{3 n+2}=P_{3 n+1} \cup$ $\left\{c_{n}=m\right\}$.

(4) If $P_{3 n+2}$ is defined, we can similarly find $P_{3 n+3} \supset P_{3 n+2}$ such that for some $m, a_{n}=m \in P_{3 n+3}$.

As in [1, Theorem 3.3] we get a generic model $N(P)$ which satisfies all our conditions.

Now let us prove $(*)$

(a) If $\psi \in T$, and not $\varnothing \mathbb{H}^{*} \psi$ then for some $P, P \Vdash \neg \psi$, so $N(P) \vDash \neg \psi$. As $\psi \in L^{*}$, and $N$ is the reduct of $N(P)$ to $L^{*}, N \vDash \neg \psi$, contradiction so $T \subset K_{1}^{f}$, and as $T$ is complete $K_{1}^{f} \cap L^{*}=T$.

(b) If $\phi\left(x_{1}, \cdots, x_{n}\right) \in \Gamma$ and not $\varnothing \mathbb{H}^{*} \phi\left(c_{i_{1}}, \cdots, c_{i_{n}}\right)\left(i_{1}, \cdots, i_{n}\right.$ are distinct) then for some $P, P \Vdash \neg \phi\left(c_{i_{1}}, \cdots, c_{i_{n}}\right)$ so $N(p) \vDash \neg \phi\left(c_{i_{1}}, \cdots, c_{i_{n}}\right)$, contradiction to the definition of $\Gamma$.

(c) Suppose $\phi\left(c_{i_{1}}, \cdots, c_{i_{n}}\right) \in K_{1}^{f}\left(i_{1}, \cdots, i_{n}\right.$ are distinct) (otherwise, we can write $\phi$ is a different way). So for every distinct natural number $m_{1}, \cdots, m_{n}, P=\left\{c_{i_{1}}=m_{1}, \cdots, c_{i_{n}}=m_{n}\right\}$ is a forcing condition. So as $\varnothing \mathbb{H}^{*} \phi\left(c_{i_{1}}, \cdots, c_{i_{n}}\right)$, also $P \mathbb{H}^{*} \phi\left(c_{i_{1}}, \cdots, c_{i_{n}}\right)$ so $N(P) \vDash \phi\left(m_{1}, \cdots, m_{n}\right)$. So $\phi\left(x_{1}, \cdots, x_{n}\right) \in \Gamma$. 
So we prove $(*)$. By [1, Theorem 6.1$]$ (and here it can be seen directly) $K^{f}=T \cup\left\{\psi\left(c_{i_{1}}, \cdots, c_{i_{n}}\right): i_{1}, \cdots, i_{n}<\aleph_{1}\right.$ are distinct,

$$
\text { and } \left.\psi\left(x_{1}, \cdots, x_{n}\right) \in \Gamma\right\} ;
$$

clearly, by the definition of $\Gamma$, for $i \neq j, c_{i} \neq c_{j} \in K^{f}$. So let $M$ be a $K$ generic model. So it is a model of $K^{f}[1$, Definitions $3.1,3.2]$ so $\|M\| \geqq$ $\left|\left\{c_{i}: i<\boldsymbol{\aleph}_{1}\right\}\right|=\boldsymbol{\aleph}_{1}>\boldsymbol{\aleph}_{0}$. Also $M$ is model complete for $K^{j}$ hence for $\bar{T}$ (by the definition of $\Gamma$ ). This contradicts Rabin [3], that any nonstandard model of $T$ has an extension which is a model of $T$ but not an elementary extension of $M$.

\section{REFERENCES}

1. J. Barwise and A. Robinson, Completing theories by forcing, Ann. Math. Logic 2 (1970), 119-142.

2. C. C. Chang, Some remarks on model theory of infinitary languages, The Syntax and Semantics of Infinitary Languages, Lecture Notes in Math., no. 72, SpringerVerlag, Berlin and New York, 1968, pp. 36-64.

3. M. O. Rabin, Diophantine equations and non-standard models of arithmetic, Proc. Internat. Congress Logic, Methodology and Philosophy of Science (1960), Stanford Univ. Press, Stanford, Calif., 1962, pp. 151-158. MR 27 \#3540.

4. J. I. Malitz and W. N. Reinhardt, Maximal models in the language with quantifier "there exist uncountably many", Pacific J. Math. (to appear).

Department of Mathematics, University of California, los Angeles, CaliFORNIA 90024

Current address: Institute of Mathematics, Hebrew University, Jerusalem, Israel 